\title{
Mean stress effect in stress-life fatigue prediction re-evaluated
}

\author{
Jan Papuga ${ }^{1, *}$, Ivona Vízková ${ }^{1}$, Maxim Lutovinov ${ }^{1}$, and Martin Nesládek ${ }^{1}$ \\ ${ }^{1}$ Department of Mechanics, Biomechanics and Mechatronics, Faculty of Mechanical Engineering, Czech Technical University in \\ Prague, Technická 4, 16607 Prague 6, Czech Republic
}

\begin{abstract}
The paper compares various methods for computing the equivalent stress amplitude for stress cycles of non-zero mean value in stress-life fatigue prediction. A set of 11 calculation methods is evaluated. In addition to formulations based on common static or fatigue properties, the Walker formula and the generalized Linear formula are included in the investigation. These two methods use an optimization routine to find the material parameters. The final response of the methods is compared and discussed. The Walker method provides a better solution. The generalized Linear method produces inferior results, i.e. the linear fit of the segment of the Haigh diagram is not an optimal solution.
\end{abstract}

\section{Introduction}

This paper focuses on the domain in fatigue life estimation that deals with evaluations of the mean stress effect (MSE) in the stress-life approach. The problem is solved without hesitation in industrial practice, because the traditional approaches (the Goodman formula and the Gerber formula) are assumed to be valid universally and in the case of the Goodman method safely. A considerable number of relatively recent works in fatigue journals have dealt with this topic, e.g. Chu [1], Wang et al [2], Kwofie [3], Dowling et al. [4], Sekercioglu et al [5] and Niesłony et al [6], if we consider only stressbased methods.

These papers reveal a clear mismatch between general practice and the results presented by researchers. The Goodman solution is found to be unreliable and conservative. The Smith-Watson-Topper (SWT) method is shown to work better, and its generalization into the Walker formula provides even better results. New, superior MSE models are often proposed, and are proved to be valid. However, the test sets used for the validation are small, consisting of less than 10 materials (Kwofie [3], Nieslony et al [6], Sekercioglu et al [5], Wang et al $[2])$.

Recent work presented by Dowling et al [4] seems to be well substantiated by the number of data sets that are evaluated. However, Dowling at al. do not cover the newer MSE models. They claim that the Walker method offers the best solution. A closer look reveals, however, that the analysis of the prediction quality is mostly based on strain-controlled experimental data, or on fatigue curves established after the specimens were initially prestrained by a larger cycle.

Our paper re-analyses some of Dowling's conclusions on the basis of our own validation data set of experiments, which should better conform to the requirements of a stress-based analysis. Multiple methods are evaluated, and conclusions are drawn. In [4], the Walker method provided the most promising features, but this was.thanks to the way in which it was optimized. Here, we optimize the generalized Linear method in a similar way, and we compare it with the Walker method. The results of this comparison allow some interesting conclusions to be drawn.

\section{Equivalent completely reversed stress amplitude}

There are two concepts for including the mean stress in a stress-based analysis. The first approach is to reduce the stress amplitude for a given mean stress. The second approach refers to the equivalent completely reversed stress amplitude as the stress amplitude, which would cause the same damage as would be caused by the pair of stress amplitude and mean stress. This second approach was selected for our investigation.

A list of the methods chosen for the analysis can be found in Table 1. Their graphic interpretation for one particular material, Ck35, is shown in Fig. 1. Many of the methods can be ranked as simple linear methods (Goodman, Half-slope, Morrow, Soderberg), while others are exponential (ASME, Bagci, Gerber). The SWT method is of another type. The Walker method is a generalization of the SWT method. The Walker method in Table 1 is an important exception in comparison with most other methods - it includes the additional Walker exponent. If the value is 0.5 , the method degenerates to the SWT parameter.

In the analyses in [4], the Walker method was optimized in the multiple linear regression over all experimental data to obtain the optimum value of the Walker exponent. It is no wonder that the final application of this optimized method led to better results than those provided by the other methods presented here,

"Corresponding author: jan.papuga@,fs.cvut.cz, papuga@pragtic.com 
where the material parameters remained fixed the way they were set by their authors.

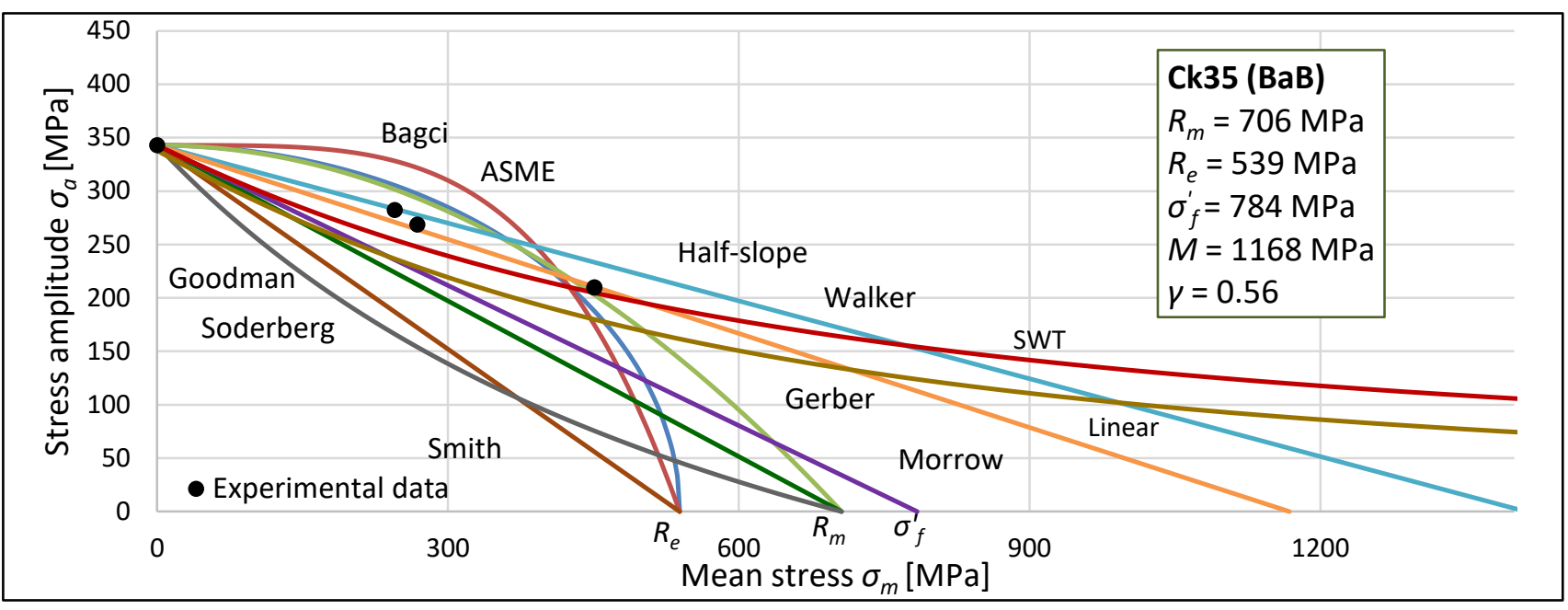

Fig. 1. Overview of various mean stress effect methods treated in this paper for the case of the BaB data set (100,000 cycles).

The complete family of linear methods differs only in one parameter, which is the stress value at which the method intersects the horizontal (mean stress) axis. These methods are among the most widely used solutions in engineering practice. The Linear method Eq. (6) was therefore prepared as a generalization of all these methods. The optimum value of its $M$ stress parameter was obtained by non-linear regression on an available experimental data in individual data sets.

Because the Walker method and the Linear method now include one optimized parameter, it is obvious that they should outperform the prediction quality of the other methods evaluated here. A more important question is, Which of these two methods will result in lower prediction errors.

\section{Composition of the data set}

As was noted in the Introduction, Dowling et al. [4] presented an extensive validation data set of experiments in their work, but many of their experimental data refer to strain-controlled experiments, and the results of some of the experiments are likely to be affected by initial overload cycles. The data set presented in Table 2 is therefore built as a new validation data set, and the following rules were applied to each partial data set:

- Only the axial loading was analysed, and a distinction was made between plane bending cases and push-pull load cases.

- Only S-N curves with more than 4 data points were admitted into the analyses.

- Each data set had to include the fully reversed axial load case.

- All S-N curves of each data set had to be prepared for a material manufactured by the same processes from the same semi-product.

- The same dimensions of the specimens (at least in the critical cross-section) were valid for all tests within each data set.

- The same environment and testing conditions had to be kept for all curves in the same data set.
Table 1. List of methods for setting the equivalent stress amplitude.

\begin{tabular}{|c|c|c|}
\hline ASME & $\sigma_{a, e q}=\frac{\sigma_{a}}{\left[1-\left(\frac{\sigma_{m}}{R_{e}}\right)^{2}\right]^{1 / 2}}$ & (1) \\
\hline Bagci & $\sigma_{a, e q}=\frac{\sigma_{a}}{1-\left(\frac{\sigma_{m}}{R_{e}}\right)^{4}}$ & (2) \\
\hline Gerber & $\sigma_{a, e q}=\frac{\sigma_{a}}{1-\left(\frac{\sigma_{m}}{R_{m}}\right)^{2}}$ & (3) \\
\hline Goodman & $\sigma_{a, e q}=\frac{\sigma_{a}}{1-\frac{\sigma_{m}}{R_{m}}}$ & (4) \\
\hline Half-slope & $\sigma_{a, e q}=\frac{\sigma_{a}}{1-\frac{\sigma_{m}}{2 \cdot R_{m}}}$ & (5) \\
\hline Linear & $\sigma_{a, e q}=\frac{\sigma_{a}}{1-\frac{\sigma_{m}}{M}}$ & (6) \\
\hline Morrow & $\sigma_{a, e q}=\frac{\sigma_{a}}{1-\frac{\sigma_{m}}{\sigma_{f}^{\prime}}}$ & $(7)$ \\
\hline Soderberg & $\sigma_{a, e q}=\frac{\sigma_{a}}{1-\frac{\sigma_{m}}{R_{e}}}$ & (8) \\
\hline Smith & $\sigma_{a, e q}=\frac{\sigma_{a} \cdot\left(1+\frac{\sigma_{m}}{R_{m}}\right)}{1-\frac{\sigma_{m}}{R_{m}}}$ & $(9)$ \\
\hline SWT & $\sigma_{a, e q}=\sqrt{\left(\sigma_{a}+\sigma_{m}\right) \cdot \sigma_{a}}$ & (10) \\
\hline Walker & $\sigma_{a, e q}=\left(\sigma_{a}+\sigma_{m}\right)^{1-\gamma} \cdot \sigma_{a}^{\gamma}$ & (11) \\
\hline
\end{tabular}

- Stress-controlled tests accepted with a minimum applicable lifetime of 10000 cycles.

- Only experiments on smooth unnotched specimens were admitted.

- Only fatigue strengths within the interpolation regions of all regression curves evaluated by the MSE method were used. 
Table 2. Composition of the data set. Explanation of notes: ${ }^{* 1}$ Finish turned, ${ }^{* 2}$ Ground, ${ }^{* 3}$ Normalized, ${ }^{* 4}$ Hardened, ${ }^{* 5}$ Longitudinal direction, ${ }^{*}$ Transverse direction, ${ }^{* 7}$ Not stated in the reference

\begin{tabular}{|c|c|c|c|c|c|c|c|c|c|c|c|}
\hline \multirow{2}{*}{$\begin{array}{l}\text { Data set } \\
\text { mark }\end{array}$} & \multirow[b]{2}{*}{ Material } & \multirow{2}{*}{$\begin{array}{c}\text { Material } \\
\text { type }\end{array}$} & \multirow{2}{*}{$\begin{array}{c}R_{m} \\
{[\mathrm{MPa}]}\end{array}$} & \multirow{2}{*}{$\begin{array}{c}R_{e} \\
{[\mathrm{MPa}]}\end{array}$} & \multirow{2}{*}{$\begin{array}{c}\sigma_{f}^{\prime} \\
{[\mathrm{MPa}]}\end{array}$} & \multirow{2}{*}{$\begin{array}{l}\text { Axial } \\
\text { load } \\
\text { mode }\end{array}$} & \multirow{2}{*}{$\begin{array}{l}\text { Applicable } \\
\text { curves with } \\
\text { MSE }\end{array}$} & \multicolumn{2}{|c|}{ N. of datapoints } & \multirow{2}{*}{$\begin{array}{c}\text { Source } \\
\text { type }\end{array}$} & \multirow[b]{2}{*}{ Ref. } \\
\hline & & & & & & & & $\begin{array}{c}\text { All } \\
\text { curves }\end{array}$ & $R=-1$ & & \\
\hline $\mathrm{BaB}$ & Ck35 & Steel & 706 & 539 & 784 & Ten & 3 & 28 & 8 & graph & [7] \\
\hline Bai & 34CrMo4 & Steel & 902 & 706 & 1022 & Ten & 3 & 35 & 12 & graph & [7] \\
\hline FAD30 & 2124-T851*1 & Al alloy & 477 & 440 & 1828 & Ten & 2 & 39 & 13 & raw & [8] \\
\hline FAD40 & $2124-\mathrm{T} 851^{* 2}$ & $\mathrm{Al}$ alloy & 477 & 440 & 1146 & Ten & 3 & 36 & 11 & raw & [8] \\
\hline Fin & 76S-T61 & Al alloy & 508 & 470 & 901 & $\mathrm{~PB}$ & 4 & 39 & 7 & graph & [9] \\
\hline GBA & 24S-T3 & Al alloy & 503 & 372 & 1963 & Ten & 4 & 100 & 43 & raw & {$[10],[11]$} \\
\hline GBB & $75 \mathrm{~S}-\mathrm{T} 6$ & Al alloy & 569 & 524 & 2286 & Ten & 4 & 53 & 10 & raw & {$[10]$} \\
\hline GBC & SAE $4130^{* 3}$ & Steel & 807 & 679 & 1142 & Ten & 3 & 31 & 5 & raw & {$[10]$} \\
\hline GBD & SAE $4130^{* 4}$ & Steel & 1241 & 1200 & 3917 & Ten & 1 & 18 & 13 & raw & [11] \\
\hline GrN & GGG-60 & Cast iron & 635 & 425 & 1109 & Ten & 1 & 28 & 19 & graph & [12] \\
\hline Han & SAE 4340 & Steel & 844 & 732 & 3611 & PB & 4 & 71 & 20 & graph & [13] \\
\hline KLN & 2017A-T4 & Al alloy & 545 & 395 & 1215 & $\mathrm{~PB}$ & 3 & 57 & 19 & raw & {$[14],[15]$} \\
\hline No40 & JIS SPV 50 & Steel & 628 & $-^{* 7}$ & 4519 & Ten & 1 & 14 & 7 & raw & {$[16]$} \\
\hline No53 & SUS304-HP & Steel & 618 & $-^{* 7}$ & 481 & Ten & 2 & 30 & 10 & raw & {$[17]$} \\
\hline Ra1 & EN-GJV-450 & Cast iron & 498 & 368 & 1158 & Ten & 5 & 147 & 29 & graph & {$[18]$} \\
\hline $\mathrm{SaL}$ & Alcoa 14S-T & $\mathrm{Al}$ alloy & 498 & 441 & 995 & $\mathrm{~PB}$ & 3 & 25 & 7 & raw & [19] \\
\hline SHM & Ck45 & Steel & 690 & 384 & 995 & Ten & 1 & 12 & 6 & raw & {$[20]$} \\
\hline $\mathrm{SiB}^{* 5}$ & $\mathrm{Ck} 45$ & Steel & 850 & 812 & 927 & Ten & 1 & 34 & 17 & graph & [21] \\
\hline $\mathrm{SiB}^{* 6}$ & $\mathrm{Ck} 45$ & Steel & 856 & 807 & 1493 & Ten & 1 & 19 & 10 & graph & {$[21]$} \\
\hline
\end{tabular}

Table 2 explicitly notes cases where the referred source failed to provide the necessary information in raw values (typically in a table), and the values had to be retrieved by a graph-reading procedure.

\section{Methods of comparison}

All the methods, with the exception of the Walker method and the Linear method, are analysed in the same way. Each fatigue curve is analysed by a linear regression, using the Basquin formula. The interpolation region of each curve is then set, and it is compared with the interpolation region of the fully reversed S-N curve. Their final intersection corresponds the applicable validation range to prevent extrapolations.

The material parameters necessary for running the methods, Eqs. (1-5, 8, 9), were available for individual materials in the referred sources. The deduction of material parameters $\gamma$ and $M$ is described below. The only remaining parameter is the $\sigma_{f}^{\prime}$ fatigue strength coefficient. This value was obtained from the Basquin regression analysis of the $\mathrm{S}-\mathrm{N}$ curve in fully reversed axial loading.

The fatigue strengths corresponding to the 1,2 and 5multiple of each decade are established, starting at 10000 . These fatigue strengths relate to a combination of the stress amplitude and the mean stress for which the equivalent stress amplitude $\sigma_{a, e q}$ is set by the methods presented in Table 1. This equivalent stress amplitude is then compared with the fatigue strength of the fully reversed cycle $\sigma_{a,-1}$ for the same lifetime. On this basis, the fatigue index error $\Delta F I$ is computed as the relative deviation from the perfect estimate:

$$
\Delta F I=\frac{\sigma_{a, e q}-\sigma_{a,-1}}{\sigma_{a,-1}}
$$

The negative values mean that an excessively low equivalent stress cycle was set, leading to a nonconservative response. A perfect estimate results in $\Delta F I=0$.

Fig. 1 shows at a glance that some of the methods must inevitably fail to result in any reasonable error. There are many cases where the mean stress value exceeds the yield stress. In these cases, none of the models using the yield stress as the material parameter could be evaluated, because the only intersection with the vertical axis would be in negative values for the Soderberg method, or it would be non-existent for the Bagci method. These cases are therefore not included in the final summary. In bending load cases, the mean stress even sometimes exceeded the tensile strength of the material. This situation can occur when there is an uneven stress distribution over the cross-section, and the material can switch to plastic behaviour. Here, too, models using tensile strength will obviously be useless.

In order to obtain some comparable results, the following limitations were set:

- Only load cases with mean stress lower than $75 \%$ of $R_{e}$ were analysed for the methods presented by ASME, Bagci and Soderberg

- Only load cases with mean stress lower than $75 \%$ of $R_{m}$ were analysed for the methods presented by Goodman, Gerber and Smith.

- Only load cases with mean stress lower than $75 \%$ of $\sigma_{f}^{\prime}$ were analysed for the Morrow method.

The output of these methods should be evaluated accordingly, because these limitations are obviously too strict for commonly observed material behaviour.

In addition to the overall statistics for the $\triangle F I$ parameter, five sub-groups were formed on the basis of material (steel, aluminium alloy, cast iron) and of the type of axial loading (plane bending, push-pull). 
In the case of the Walker method and the Linear method, linear and non-linear regression analyses were run directly on experimental data for all applicable fatigue curves in the partial data set, i.e. without involving regression analysis in the process. The regression analysis included the fatigue curve in fully reversed loading.

Using the Basquin formulation for the S-N curve:

$$
\sigma_{a, e q}=A N^{b}
$$

the Walker formula Eq. (11) was transformed by logarithms into a multiple linear formulation, as Dowling et al. suggested in [4]:

$$
\log N=\frac{1}{b}\left[\log \sigma_{\max }+\gamma \cdot \log \left(\frac{1-R}{2}\right)-\log \mathrm{A}\right]
$$

This process was also implemented on the Linear method Eq. (6), and leads to the non-linear formulation:

$$
\log N=\frac{1}{b}\left[\log \sigma_{a}+\log \frac{M}{A}-\log \left(M-\sigma_{m}\right)\right]
$$

The main purpose of this process was to obtain parameters $\gamma_{3}$ and $M_{3}$ (the subscript refers to the method by which the values are obtained - here by the optimization routine). The minimized parameter was the sum of squares of the logarithms of the cycles.

\section{Results, and a discussion}

The type of analysis favours more sophisticated optimized methods. The final results depicted in Table 3 are therefore discussed separately for optimized methods and non-optimized methods.

\subsection{Non-optimized methods}

Unlike the two optimized methods, the intersection of the functions with the horizontal axis is fixed by these non-optimized methods (with the exception of the SWT method, where there is no intersection). This property enforced the removal of some data items from the evaluation, when the mean stress was too close to the intersection point (see Sec. 4). All these methods would otherwise result in extensive errors, which would disallow any reasonable analysis.

Apart from limitation by $R_{e}, R_{m}$ or $\sigma_{\mathrm{f}}^{\prime}$ and the exception of the SWT method, as mentioned above, only one other method is available: the Half-slope method. Here, the Half-slope method has the best score. Sec. 5.4 will show the reason for its best performance, mostly for steels loaded in push-pull, and its closest similarity with the generalized Linear method.

The exponential rules (ASME, Bagci, Gerber) use a similar intersection with the mean stress axis, but they lie higher in the area of modest mean stresses than most linear methods. Their general response is therefore nonconservative. However, the trend toward high mean stresses again cannot be correct, because the material response quite frequently exceeds $R_{e}$ in the mean stresses.

The Smith method is not suitable for any evaluated sub-group, and it can be removed from any further attempts at an improvement. However, the SWT parameter, which does not contain any material parameter at all, acts almost miraculously in comparison with other non-optimized parameters, and above all its good performance for aluminium alloys should be highlighted.

\subsection{A comparison between optimized methods}

In addition to the statistical summary presented in Table 3 , histograms of the $\Delta F I$ errors are depicted in Fig. 2 (left) for the Linear method and in Fig. 2 (right) for the Walker method. In both cases, the $M_{3}$ and $\gamma_{3}$ parameters were retrieved by the optimization routine on all available experimental data, as described above.

Table 3. Overall comparison of statistical evaluations of individual methods. Note that only the SWT, Walker, Linear and Half-slope

\begin{tabular}{|c|c|c|c|c|c|c|c|c|c|c|c|c|c|c|c|c|}
\hline & \multicolumn{8}{|c|}{ Average fatigue index error $\Delta F I[\%]$} & \multicolumn{8}{|c|}{ Standard deviation $\Delta F I[\%]$} \\
\hline & \multicolumn{3}{|c|}{ Plane Bending } & \multicolumn{4}{|c|}{ Tension } & \multirow{2}{*}{ Total } & \multicolumn{3}{|c|}{ Plane Bending } & \multicolumn{4}{|c|}{ Tension } & \multirow{2}{*}{ Total } \\
\hline & $\mathrm{Al}$ & St & Total & $\mathrm{Al}$ & $\mathrm{C}$ & $\mathrm{St}$ & Total & & $\mathrm{Al}$ & $\mathrm{St}$ & Total & $\mathrm{Al}$ & $\mathrm{C}$ & St & Total & \\
\hline ASME & -13.4 & 23.7 & -6.9 & -23.4 & -7.1 & -4.8 & -13.0 & -11.3 & 8.6 & 15.3 & 17.3 & 13.2 & 33.0 & 7.6 & 18.8 & 18.6 \\
\hline Bagci & -17.1 & 16.0 & -11.3 & -26.7 & -11.5 & -10.8 & -17.6 & -15.9 & 7.6 & 15.5 & 15.7 & 14.1 & 32.8 & 8.0 & 18.6 & 18.1 \\
\hline Gerber & -8.0 & 35.3 & -0.4 & -19.1 & -10.7 & -2.8 & -10.6 & -8.1 & 12.6 & 22.1 & 22.0 & 12.1 & 32.0 & 8.0 & 17.6 & 19.2 \\
\hline Goodman & 23.2 & 103.9 & 37.3 & 9.4 & 1.8 & 33.6 & 18.6 & 23.1 & 33.7 & 49.9 & 47.7 & 21.1 & 12.2 & 16.7 & 22.3 & 31.3 \\
\hline Half-slope & -5.8 & 42.0 & 5.2 & -18.9 & -22.2 & 1.3 & -11.4 & -7.1 & 9.6 & 16.6 & 23.3 & 12.4 & 25.3 & 9.0 & 18.3 & 20.9 \\
\hline Linear $\mathbf{M}_{3}$ & -1.4 & 1.7 & -0.6 & -4.3 & 1.8 & 3.1 & 0.0 & -0.2 & 6.0 & 2.9 & 5.5 & 11.6 & 16.3 & 5.9 & 11.5 & 10.2 \\
\hline Morrow & -5.1 & 15.0 & 0.4 & -26.0 & -23.8 & 19.1 & -7.4 & -5.3 & 9.7 & 5.5 & 12.6 & 14.6 & 27.6 & 17.7 & 29.0 & 25.8 \\
\hline Soderberg & 36.6 & 151.2 & 56.6 & 18.9 & 18.1 & 62.7 & 36.7 & 42.0 & 46.5 & 86.0 & 69.7 & 27.9 & 24.0 & 33.7 & 36.7 & 48.3 \\
\hline Smith & 68.5 & 209.2 & 93.1 & 51.1 & 24.7 & 85.0 & 61.2 & 68.8 & 70.8 & 100.5 & 92.7 & 48.7 & 33.8 & 37.6 & 47.3 & 62.5 \\
\hline SWT & 6.6 & 36.6 & 14.8 & -1.5 & -6.6 & 14.8 & 4.1 & 7.0 & 10.3 & 9.2 & 16.8 & 7.7 & 10.3 & 9.8 & 12.9 & 14.8 \\
\hline Walker $\gamma_{3}$ & 1.9 & 3.0 & 2.2 & 2.6 & 0.5 & 4.5 & 2.9 & 2.7 & 4.9 & 2.4 & 4.4 & 5.4 & 9.6 & 5.8 & 6.8 & 6.2 \\
\hline Walker $\gamma_{4}$ & 8.6 & 17.3 & 11.0 & 0.8 & - & -1.5 & -0.4 & 3.3 & 11.1 & 3.3 & 10.4 & 7.9 & - & 8.6 & 8.3 & 10.4 \\
\hline
\end{tabular}
methods also present results for high mean stresses, as described in Sec. 4. Al aluminium alloys, St $\sim$ steels, $\mathrm{C} \sim$ cast irons. The coloured backgrounds highlight the best results ( $\triangle F I$ from -5 to $5 \%$ in the average, and less than $10 \%$ in the standard deviation). 


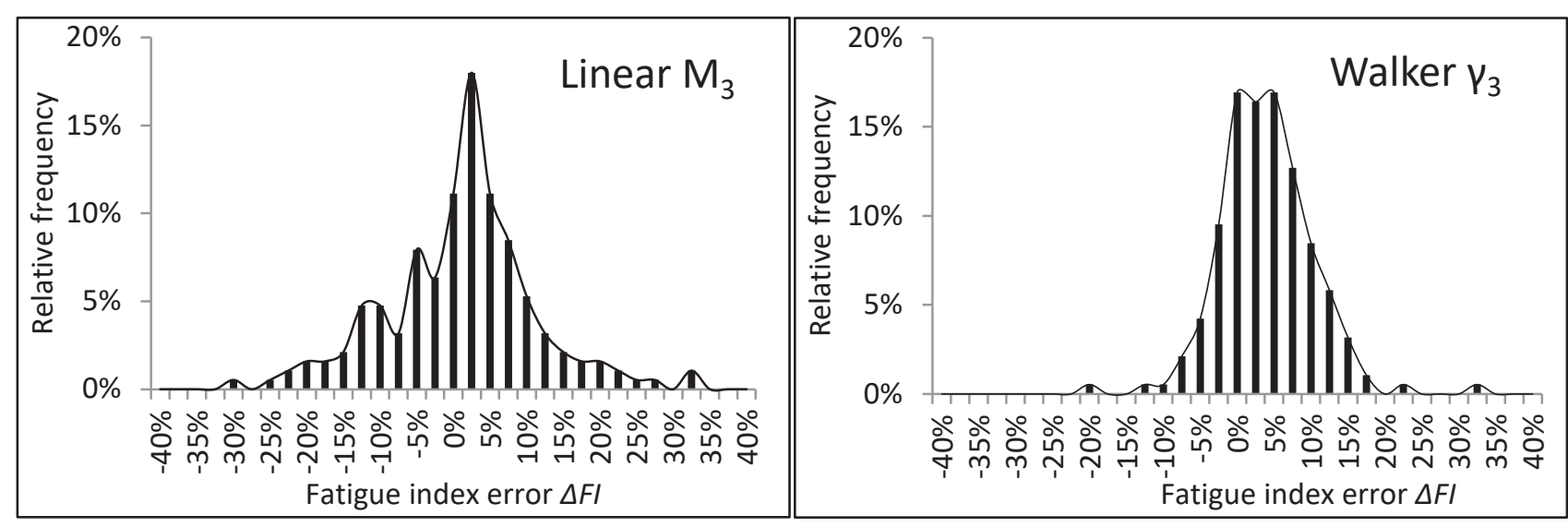

Fig. 2. (left) Histogram of the results of the Linear method, (right) Histogram of the results of the Walker method.

Though both methods contain one optimized parameter, the prediction quality of the Walker method exceeds the prediction quality that can be retrieved from the Linear method. The bottom of the histogram is not so broad when the Walker method is used. Only $71.4 \%$ of all data items lie within the range of $\pm 10 \%$ for the Linear method, in comparison with $87.3 \%$ when the Walker solution is applied. These levels would change to $93.1 \%$ for the Linear method and $98.4 \%$ for the Walker method, if the allowed error range is raised to $\pm 20 \%$.

An additional histogram presented in Fig. 3 was prepared for a very special case designed as a "worst case scenario". While the optimum Walker parameter is obtained by best-fit analysis over a number of fatigue curves, most engineers are not so well equipped with data. A typical best-case situation in practice is if there is some curve including the mean stress effect in addition to the fully reversed case.

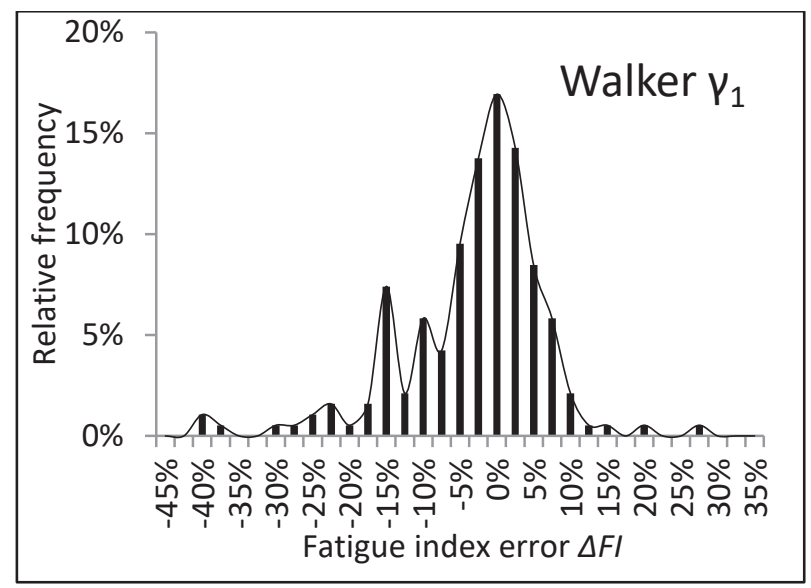

Fig. 3. Histogram of all results for the worst-case scenario for the Walker method.

The worst-case analysis is derived from a regression analysis of just two curves, one of which corresponds to fully reversed loading, while the other corresponds to any other curve with non-zero mean stress. From all such combinations within each partial data set, the combination leading to the maximum $\gamma_{1}$ value is selected (subscript 1 is added to distinguish it from other cases). A search is made for the maximum value, because the Walker formulation then leads to the smallest equivalent stress value and to the most non-conservative prediction.
Due to this intentional selection of the unsafest cases, the output is no longer either symmetrical or Gaussian, and the median value is shifted more to the nonconservative side. However, if the content of the $\pm 10 \%$ and $\pm 20 \%$ intervals is checked, the resulting values are $75.1 \%$ for $\pm 10 \%$ and $93.7 \%$ for $\pm 20 \%$, i.e. the values obtained by the best-fit optimization procedures of the Linear method. We can conclude that the use of the Walker method with the exponent tuned to any additional experimental data, including the mean stress effect, leads to results that are at worst similar in quality to those produced by any of the linear methods.

\subsection{Estimates of Walker parameter $\gamma$}

Dowling et al. in [4] proposed estimates of $\gamma$ exponents for steels and aluminium alloys. Constant values were set for aluminium alloys: 0.651 for low-strength alloys, and 0.473 for high-strength alloys. For steels, the function

$$
\gamma=-0.0002 \cdot R_{m}+0.8818
$$

was set. These proposals are confronted in Figs. 4-5; all values are presented in Fig. 6.

Regression models would lead to slightly different values and formulas, but the differences are not excessive. Scarce evidence from a limited number of tested data sets suggests that it does not make sense to provide an improved formula. This is particularly evident for steels presented in Fig. 4, where only a single high-strength steel serves as a clue to the material behaviour.

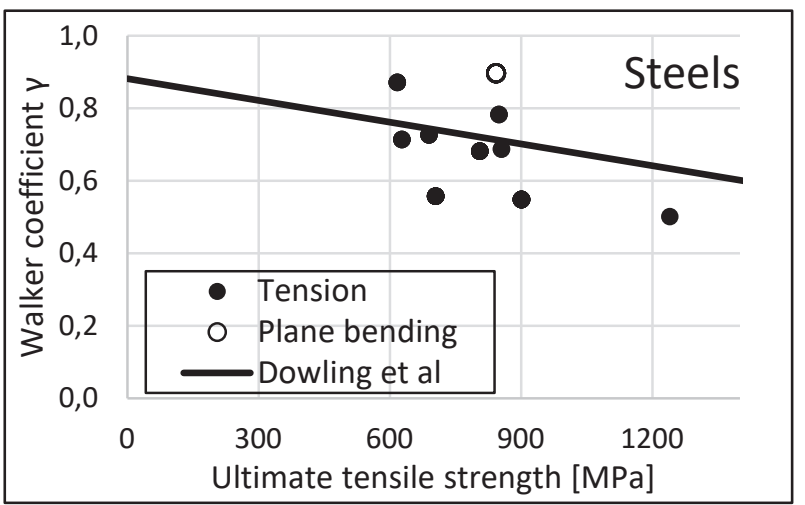


Fig. 4. A comparison between the proposal of Dowling et al. [4] for an estimate of the Walker exponent with the best-fit parameters from the experiments on steels.

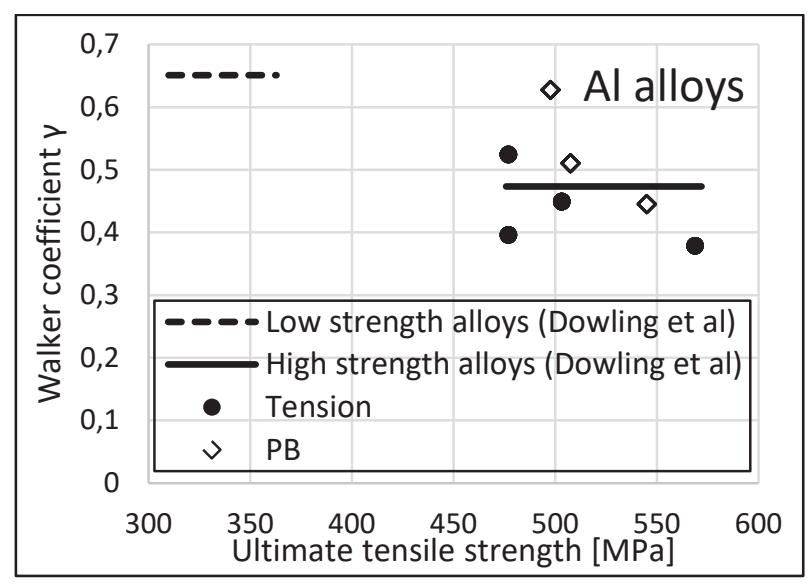

Fig. 5. A comparison of the proposals of Dowling et al. [4] for an estimate of the Walker exponent with the best-fit parameters from the experiments on aluminium alloys.

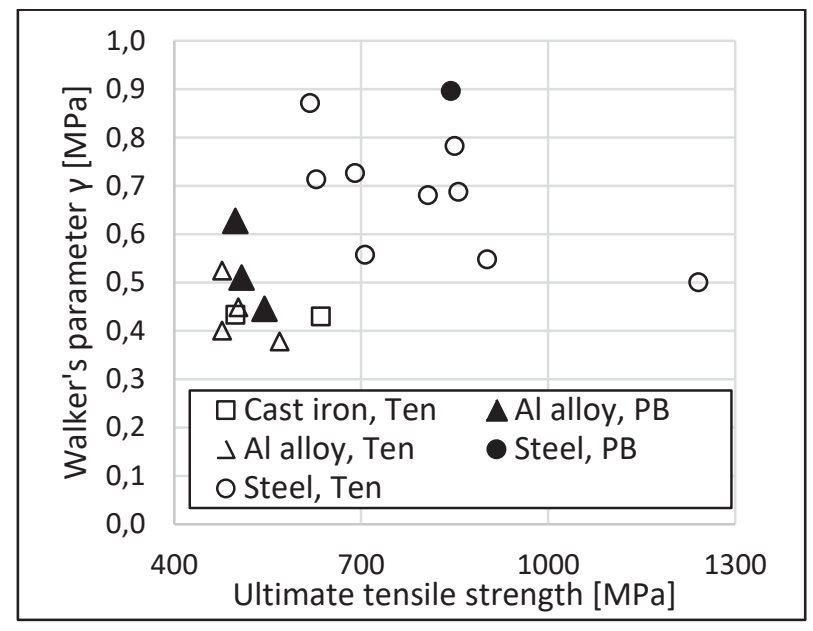

Fig. 6. Dependency of the $\gamma$ exponent of the Walker method on the tensile strength. Ten $\sim$ push-pull, $\mathrm{PB} \sim$ plane bending.

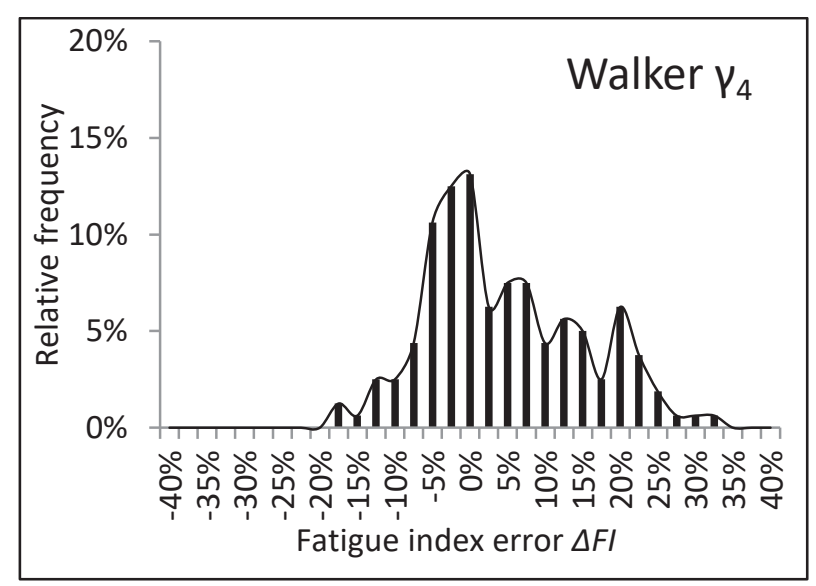

Fig. 7 Histogram of fatigue index errors for the Walker method and the $g$ exponents retrieved by the proposal by Dowling et al. [4]. Note that only steels and aluminium alloys are evaluated.
The use of the coefficients proposed by Dowling et al. on the validation set resulted in the histogram presented in Fig. 7. Subscript 4 is added to the Walker exponent to distinguish it from other solutions aimed at deriving the exponent. The histogram is much lower than the histograms presented above, and it is more spread to conservative values of the prediction. If the content of the $\pm 10 \%$ and $\pm 20 \%$ intervals is checked, values of $66.3 \%$ for $\pm 10 \%$ and $92.5 \%$ for $\pm 20 \%$ are obtained. This makes the prediction more dispersed than when the generalized Linear method is applied.

The range of the $\gamma$ exponent from 0.5 to 0.9 for steels does not seem to be significantly affected by the value of $R_{m}$ - there is only a weak relation (see Fig. 6). Nevertheless, even the use of the proposed relation is better than the straightforward use of $\gamma=0.5$, as in the SWT method (see Table 3 - compare the results for the optimized Walker method $\gamma_{3}$ with the proposal of Dowling et al $\gamma_{4}$ and SWT). Fig. 4 shows that the SWT method has to be generally safe for steels, because $\gamma=0.5$ intrinsic to the SWT method forms the lower envelope of the optimized parameters, which results in the maximum mean stress effect.

For the high-strength aluminium alloys depicted in Fig. 5, the best-fit results are gathered close to a value of 0.5. This can lead to the conclusion that even the SWT method is a good fit for the general MSE behaviour for this material group.

\subsection{M parameter of the Linear method}

A similar comparison between the optimized $M$ parameter and the tensile strength is presented in Fig. 9. The quadrant axis depicted in the figure shows that the expectation $M=R_{m}$ used by the Goodman method is generally too conservative. It also shows that the Halfslope formula crosses the cluster of points much better.

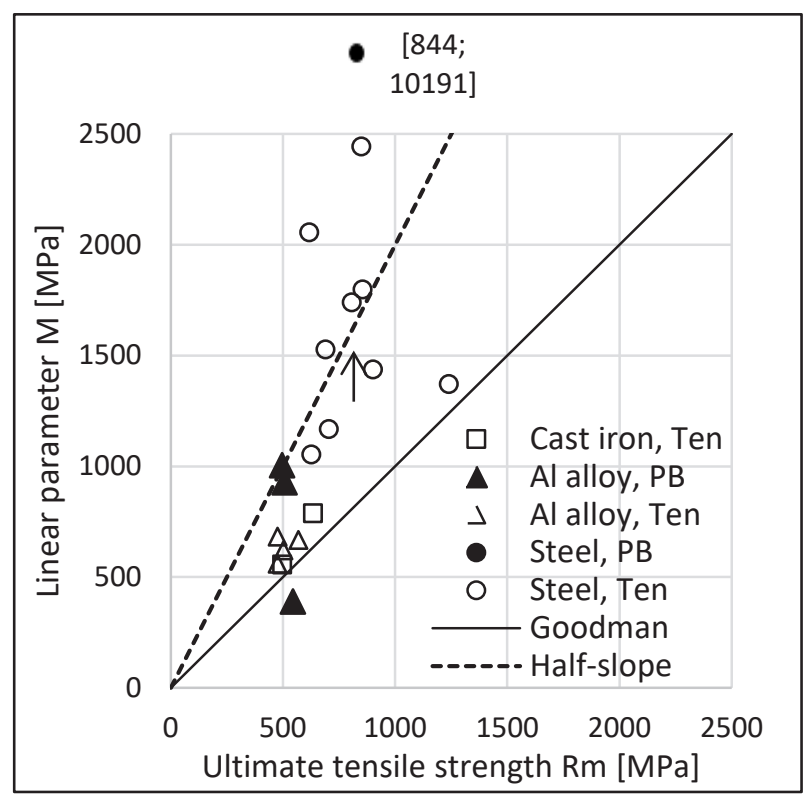

Fig. 9. Dependency of the $M$ parameter of the Linear method on tensile strength. Ten $\sim$ push-pull loading, $\mathrm{PB} \sim$ plane bending. 
Fig. 10 was prepared in order to provide a better overview of how well each of the linear methods corresponds with the optimum Linear method. The ratios shown in the figure refer to various methods presented here:



Fig. 10. A comparison between the optimized $M$ parameter and other constants of linear methods. Ten $\sim$ push-pull, $\mathrm{PB} \sim$ plane bending.

Table 4. The sum of squares of the relative errors from ideal values in the four items in the list above, as evaluated on the individual data sets depicted in Fig. 10. Al aluminium alloys, $\mathrm{St} \sim$ steels, $\mathrm{C} \sim$ cast irons.

\begin{tabular}{|c|c|c|c|c|c|c|c|c|}
\cline { 2 - 8 } \multicolumn{1}{c|}{} & \multicolumn{3}{c|}{ Plane Bending } & \multicolumn{4}{c|}{ Push-pull } & \multirow{2}{*}{ Total } \\
\cline { 2 - 8 } \multicolumn{1}{c|}{} & Al & St & Total & Al & C & St & Total & \\
\hline Goodman & 1.3 & 11.1 & 11.2 & 0.5 & 0.3 & 3.8 & 3.8 & 11.8 \\
\hline Half-slope & 1.3 & 10.1 & 10.2 & 1.5 & 1.2 & 1.9 & 2.7 & 10.5 \\
\hline Morrow & 0.7 & 1.8 & 1.9 & 1.3 & 0.6 & 3.9 & 4.2 & 4.6 \\
\hline Soderberg & 1.6 & 12.9 & 13.0 & 0.9 & 1.0 & 4.4 & 4.6 & 13.8 \\
\hline
\end{tabular}

- The Goodman method would fit with $M / R_{m}=1$

- The Half-slope method corresponds to $M / R_{m}=2$

- Morrow method $\sim M / \sigma_{f}^{\prime}=1$

- Soderberg method $\sim M / R_{e}=1$

It should be noted that the Soderberg formula using the yield stress is not optimal, even for the brittle materials (cast irons here) for which it is usually proposed. The use of $R_{m}$ is generally conservative, and the $M / R_{m}$ ratio of 2 is obviously a closer estimate.

Table 4 was prepared to improve the comprehensibility of Fig. 10, and to quantify the differences between these four methods and a perfect prediction. For each data set, Table 4 presents the sum of squares of the absolute deviations from the equalities defined in the list above. The values presented in Table 4 are obviously extremely affected by the plane bending case of the Han data set. Because the Han set is what produces the enormous differences, Table 5 was prepared without this test set. Table 5 shows that, when the Han data set is removed, the Half-slope method presents the smallest deviation from the optimum value.

There is a simple answer to the question, Why is the Han data set well modelled only by the Morrow method?
It is caused by the way in which the $\sigma_{f}^{\prime}$ parameter was defined. It was not obtained from the hysteresis loop in the push-pull test, but as the best fit to the fully reversed plane bending loading. The stress distribution effect, and the induced plastic redistribution, are similar for all S-N curves in this test set. However, parameters $R_{m}$ and $R_{e}$, which were used in the analyses by other methods, were not impacted at all by the stress distribution effect. This case illustrates very clearly how dubious it is to include plane bending cases in these analyses, because the stress gradient effect is involved.

Table 5. The sum of squares of the relative errors from the perfect values as in Table 4, but without the Han data set.

\begin{tabular}{|c|c|c|c|c|c|c|}
\cline { 2 - 7 } \multicolumn{1}{c|}{} & PB & \multicolumn{4}{c|}{ Push-pull } & \multirow{2}{*}{ Total } \\
\cline { 2 - 7 } \multicolumn{1}{c|}{} & Al & Al & C & St & Total & \\
\hline Goodman & 1.3 & 0.5 & 0.3 & 3.8 & 3.8 & 4.0 \\
\hline Half-slope & 1.3 & 1.5 & 1.2 & 1.9 & 2.7 & 3.0 \\
\hline Morrow & 0.7 & 1.3 & 0.6 & 3.9 & 4.2 & 4.2 \\
\hline Soderberg & 1.6 & 0.9 & 1.0 & 4.4 & 4.6 & 4.9 \\
\hline
\end{tabular}

Table 5 shows that the Goodman method is not far from the optimum values for aluminium alloys and cast irons. For steels, the Half-slope formula achieves the best results. Nevertheless, these conclusions should be taken only with care, because there is a substantially bigger number of items in the steel group than in the aluminium alloy and cast iron groups.

\section{Conclusion}

This paper has presented a comparison of various methods for computing the equivalent stress amplitude for a given mean stress value. In comparison with most 
methods of fixed common material parameters, the Walker method and the generalized Linear formula were treated separately, because values of their additional parameters were optimized.

The following major conclusions can be drawn:

1. The Half-slope formula provides the best results for simple non-optimized solutions based on static material parameters.

2. The SWT method is even better, and its performance for aluminium alloys is close to the optimum values provided by the optimized Walker method.

3. A comparison of the optimized formulas indicated that the Walker method leads to a better solution than the generalized Linear formula.

4. The dependency of the Walker exponent on the tensile strength proposed by Dowling et al. in [4] is reasonable, though the dependency is quite weak. Direct use of this parameter results in a scatter of the results similar to that provided by the optimized Linear method. These estimates provide better prediction results than widely-used SWT.

5. If there is any additional S-N curve with non-zero mean stress to accompany the S-N curve with fully reversed loading, the use of non-linear regression to obtain the optimum Walker's exponent is likely to result in a better prediction than the use of the fully optimized Linear method, or at least an equally good prediction.

6. All methods, with the exception of the optimized methods, SWT method an Half-slope method, had to be analysed only for cases where the mean stress was lower than $75 \%$ of their intersection with the mean stress axis (be it tensile strength, yield stress or fatigue strength coefficient). For higher mean stresses, these methods are meaningless.

7. The plane bending load case is affected by the nonuniform stress distribution. If it is necessary to use one of the simpler methods, on which the $75 \%$ limit noted above must be imposed, the plane bending load effect should be treated separately.

Acknowledgements: The authors thank the Ministry of Education, Youth and Sport and the Czech Technical University in Prague for its financial support for the research presented here within the framework of grant No. SGS17/175/OH K2/3T/12.

\section{References}

1. C.-C. Chu, In: SAE 2000 World Congress. SAE International, Detroit (2000)

2. S. Wang, M. Dixon, C.O. Huey, S. Chen, Journal of Mechanical Design. 122, 143-146 (2000)

3. S. Kwofie, Int Jnl of Fatigue. 23, 829-836 (2001)

4. N. Dowling, C. Calhoun, A. Arcari, Fat \& Fract of Engng Maters \& Structs. 32, 163-179 (2009)

5. T. Sekercioglu, Materialwissenschaft und Werkstofftechnik. 40, 713-717 (2009)

6. A. Niesłony, M. Böhm, Int Jnl of Fatigue. 52, 49-56 (2013).
7. F. Baier, Zeit- und Dauerfestigkeit bei überlagerter statischer und schwingender Zug-Druck- und Torsionbeanspruchung. (Universität Stuttgart, Stuttgart 1970)

8. J. Papuga, F. Fojtík, M. Vargas, A. Hodr, A. Karolczuk, M. Fusek, R. Halama, Summary of experiments on 2124-T851 realized within FADOFF project. [FAD/14/001]. (CTU in Prague, Prague 2014)

9. W.N. Findley, Combined-stress fatigue strength of 76S-T61 aluminum alloy with superimposed mean stresses and corrections for yielding. [NACA TN2924]. (NACA, Washington, 1953)

10. H.J. Grover, S.M. Bishop, L.R. Jackson, Fatigue strengths of aircraft materials: Axial-load fatigue tests on unnotched sheet specimens of 24S-T3 and 75S-T6 aluminum alloys and of SAE 4130 steel. [NACA TN 2324]. (NACA, Washington, 1951)

11. W. Illg, Fatigue tests on notched and unnotched sheet specimens of 2024-T3 and 7075-T6 aluminum alloys and of SAE 4130 steel with special consideration of the life range from 2 to 10,000 cycles. [NACA-TN-3866]. (NACA, Washington, 1956)

12. V. Grubisic, J. Neugebauer, Giessereiforschung 31, 123-128 (1979)

13. B.C. Hanley, The effect of range of stress and state of stress on the fatigue strength of SAE 4340 steel. (University of Illinois Urbana, Illinois, 1951)

14. K. Kluger, T. Łagoda, Int Jnl of Fatigue 66, 229-245 (2014).

15. A. Niesłony, T. Łagoda, K. Walat, M. Kurek, Materialwissenschaft und Werkstofftechnik 45, 947952 (2014)

16. Data sheets on fatigue properties for butt welded joints of SPV50 steel plate for pressure vessels, Effect of stress ratio. [NRIM Fatigue Data Sheet No. 40]. (National Research Institute for Metals, Tokyo, 1984)

17. Data sheets on fatigue properties for butt welded joints of SUS304-HP (18Cr-8Ni) hot rolled stainless steel plate, Effect of stress ratio. [NRIM Fatigue Data Sheet No. 53]. (National Research Institute for Metals, Tokyo, 1986)

18. T. Rausch, Zum Schwingfestigkeitsverhalten von Gusseisenwerkstoffen unter einachsiger und mehrachsiger Beanspruchung am Beispiel von ENGJV-450. (Shaker Verlag, Aachen, 2011)

19. M. Shariati, H. Mehrabi, Fat \& Fract of Engng Maters \& Structs 38, 489-502 (2015)

20. A. Simbürger, Festigkeitsverhalten zäher Werkstoffe bei einer mehrachsigen phaseverschobenen Schwingbeanspruchung mit körperfesten und veränderlichen Hauptspannungsrichtungen. (TH Darmstadt, Darmstadt 1975)

21. J.A. Sauer, D.C. Lemmon, Transactions of the American Society for Metals 42, 559-576 (1950). 